\title{
Joint Power Control and User Association for Traffic Offloading in Heterogeneous Networks
}

\author{
Po-Han Chiang ${ }^{1}$, Po-Han Huang ${ }^{1}$, Shi-Sheng Sun ${ }^{2,3}$, Wanjiun Liao ${ }^{1,2}$, and Wen-Tsuen Chen $^{3}$ \\ ${ }^{1}$ Graduate Institute of Communication Engineering, National Taiwan University, Taipei, Taiwan \\ ${ }^{2}$ Department of Electrical Engineering, National Taiwan University, Taipei, Taiwan \\ ${ }^{3}$ Institute of Information Science, Academia Sinica, Taipei, Taiwan \\ Email: \{pchiang,r00942032,d96921023,wjliao\}@ntu.edu.tw, chenwt@iis.sinica.edu.tw
}

\begin{abstract}
A heterogeneous network (HetNet), which consists of macro cells and small cells, is an effective tool to improve the system spectral efficiency by exploiting the benefit of spatial reuse. However, the benefit of HetNet is limited by the asymmetry of transmit power between macro base stations (BSs) and pico BSs. Traditional solutions lead to a tradeoff between received signal strength (RSS) and load balancing. To tackle this problem, we propose a novel mechanism with joint macro BS transmit power control and load-aware user association. As such, we can realize traffic offloading from the macro cell to pico cells in a two-tier HetNet and mitigate intercell interference (ICI) in the network. We formulate this problem as a nonlinear integer programming problem, and prove that it is NP-hard. We then propose a polynomial-time heuristic. The simulation results show that our algorithm is indeed an efficient mechanism with better network performance in terms of average throughput and fairness.
\end{abstract}

Keywords-- HetNet, traffic offloading, power control, frequency reuse

\section{INTRODUCTION}

With the popularity of smart phones and the high data rate requirements for many applications, mobile data traffic is expected to grow exponentially. Cell size reduction to reuse the spectrum is the simplest and most effective way to enhance network capacity [1]. Deploying heterogeneous networks (HetNet), comprising both traditional BSs (macro BSs) and low power nodes such as pico BSs, is the most promising solution to increase the spatial spectrum efficiency (bps/Hz/area), enhance the total network capacity, and provide superior user experience [2][3].

However, the benefits of HetNet are limited by the asymmetry of transmit power between macro BSs and low power nodes since users (UEs) always associate with the BS based on the strongest reference signal receiving power (RSRP) in traditional cellular networks. Therefore, the coverage of low power nodes shrinks in dense interferencelimited networks, which results in underutilization of low power nodes [3]. The co-channel deployment in HetNet further exacerbates the inter-cell interference (ICI) due to the near-far effect. These problems are shown in Fig. 1.

In LTE, cell range expansion (CRE), which virtually adds a bias value of RSRP to pico BSs, is proposed to extend coverage of pico cells [4]. The main challenges of CRE include: $i$ ) UEs at the edge of pico cells are severely interfered by macro BSs and suffer poor downlink Signal to

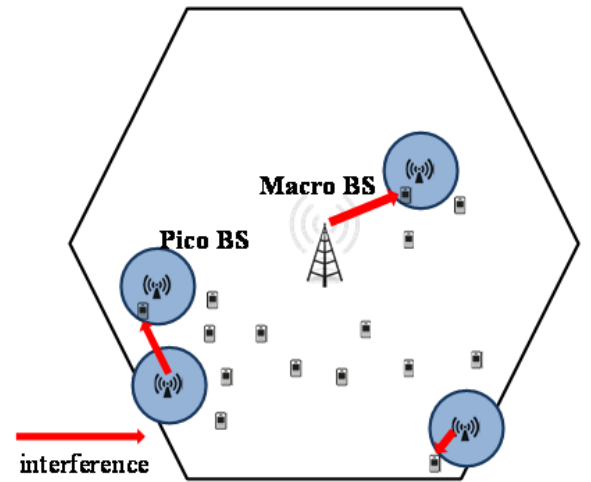

Fig. 1. Underutilization and the ICI problem in HetNet. The coverage of pico BSs shrinks due to the interference from macro $\mathrm{BS}$ and the arrows represent the interference path among BSs.

Interference plus Noise Ratio (SINR). ii) A predefined bias value is not suited for all users in the network, since the optimal value may vary with the geographical distribution of users, and the traffic load of BSs [5].

In addition to adding bias to the RSRP of low power nodes, traffic offloading can be achieved by adjusting the transmit power of macro BS. The so-called "cell breathing" technique [6] shifts the cell boundary by adjusting their transmit power in homogeneous network. However, to realize the power control in HetNet, two unique issues need to be addressed: $i$ ) the coverage of macro BS should still be guaranteed even when the transmit power is reduced, and $i$ ) UEs in macro cells may benefit from traffic offloading at the cost of the degraded SINR, thus the decision of the optimal transmit power level is important. Moreover, user association should be based on both RSRP and the load information of BSs since the throughput of users depends on both channel conditions and available time and frequency resources.

In [5][7], the authors focus on coordinating the transmit power and resource allocation among BSs to mitigate ICI in HetNet with CRE. The authors in [8] propose a fractional frequency reuse and power control scheme to improve the long term throughput. In above studies, the load condition of $\mathrm{BSs}$ is rarely discussed. Besides, in these strategies, user association is defined according to a fixed CRE bias, and the optimal user association strategies are yet to be investigated. 
In [9][10], dynamic user association methods are proposed to balance the loads among BSs and to deal with the tradeoff between performance and fairness. However, these works are designed for homogeneous networks and are not easily extended to HetNet. The work in [11] is one of the few study on user association in HetNet, but it does not discuss the impact of transmit power control on user association.

In this paper, we study the joint power control and user association problem for traffic offloading in HetNet. To improve the system capacity and to preserve the coverage, a novel two-tier power control scheme together with a new frequency reuse scheme is proposed. We formulate this problem as a nonlinear integer programming problem, and prove it is NP-hard. We then propose an iterative power control mechanism and load-aware user association which jointly considers the channel condition and load information to optimize the network throughput. The simulation results show that our scheme is indeed effective.

The rest of the paper is organized as follows. In Sec. II, the network model and the problem are described. In Sec. III, the problem is formulated as a nonlinear integer linear programming problem. The proposed power control and user association algorithm is described in Sec. IV. In Sec. V, the performance of the proposed mechanism is evaluated via simulations. Finally, the paper is concluded in Sec. VI.

\section{SYSTEM ARCHITECTURE}

In this paper, we focus on downlink two-tier cellular networks with multiple pico BSs in each macro cell.

1) The downlink bandwidth is divided into sub-bands and shared by the macro BS and pico BSs. Each BS schedules its users to sub-bands.

2) The transmit power of the macro BS on each sub-band can be adjusted separately as in [5][7], and the transmit power of pico BSs is fixed for practical reasons.

3) The load information among the macro and pico BSs can be exchanged via the high speed X2 interface or the Operations, Administration, Maintenance (OAM) procedure.

4) Each user is only associated with one BS (either macro or pico) at any time and a full buffer traffic model is assumed. Users estimate the channel conditions by measuring the pilot signal and reporting back to their serving BSs.

\section{A. Propagation Model}

The propagation channel depends on the physical environment. Here we consider a basic channel model with path loss. Specifically, the receive power of an UE, denoted by $P_{r}$, is expressed as

$$
P_{r}=\Gamma P_{t},
$$

where $P_{t}$, is the transmit power, and $\Gamma$ is the channel gain between transmitter and receiver and is represented as:

$$
\Gamma=\frac{g_{t} g_{r}}{\delta_{t r}},
$$

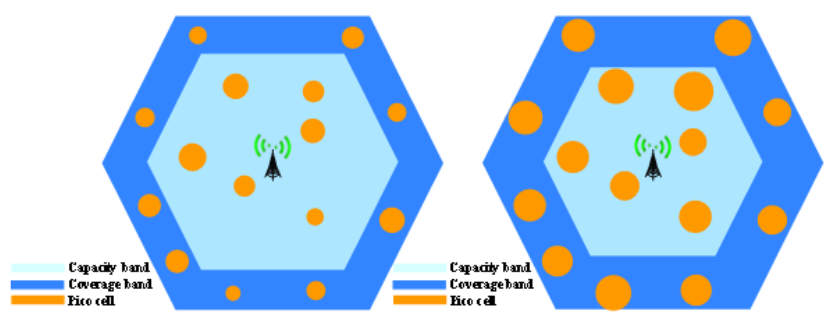

(a)

(b)

Fig. 2. Traffic offload from macro BS to pico BSs (a) Macro BS transmits at the maximum power level (b) The coverage of pico BSs expands since macro BS reduces the transmit power on the capacity band.

where $g_{t}, g_{r}, \delta_{t r}$ denote the transmitter antenna gain, receiver antenna gain, and path loss between transmitter and receiver, respectively.

\section{B. User Traffic Model}

The instantaneous achievable link rate of UE $k$ in the cell of $\mathrm{BS} b$ is derived as follows:

$$
r_{k i}=T_{k i} w_{k i},
$$

where $w_{k i}$ and $T_{k i}$ denote the given bandwidth and spectral efficiency of UE $k$ served by BS $i$, respectively. We define the corresponding $S I N R_{k i}$ and the spectral efficiency $T_{k i}$ of UE $k$ connected to $\mathrm{BS} i$ from the Shannon equation as follows:

$$
\begin{gathered}
S I N R_{k i}=\frac{P_{k i}}{\sum_{j \in \mathrm{B} \backslash\{i\}} P_{k j}+\sigma^{2}}, \\
T_{k i}=\log _{2}\left(1+S I N R_{k i}\right),
\end{gathered}
$$

where $P_{k i}$ represents the received power of UE $k$ from BS $i$ and $\sigma$ is the noise power level on the given bandwidth.

Assuming each BS schedules its users independently, we can obtain the long-term average throughput of UE $k$ using similar procedures as in [9]:

$$
R_{k}=\frac{G\left(Y_{i}\right) r_{k i}}{Y_{i}}
$$

where $Y_{i}$ is the total number of UEs served by BS $i$ and $G\left(Y_{i}\right)$ represents the long-term scheduling gain, which will be explained in (15).

\section{Power Control Mechanism}

Since the throughputs of UEs depend on their channel conditions and available time/frequency resources, each macro BS can adjust its transmit power to achieve better balance between offloading more UEs to pico BSs and the SINR of its users. On the other hand, the coverage may not be ensured if the transmit power of the macro BS is reduced and the pico BSs are not properly deployed.

In our mechanism, the problem can be solved by dividing the available bandwidth of each macro BS into two bands: the coverage band and the capacity band. The transmit power is adjusted only on the capacity band while the power on the coverage band is fixed to serve the users located in 
potential coverage holes. For example, if the traffic load of a macro cell is high, the macro BS can reduce its transmit power so that more users can be served by the pico BSs with guaranteed coverage on the coverage band, as shown in Fig. 2. In this paper, a proper frequency partitioning is assumed based on the statistics collected among cells.

\section{PROBLEM FORMULATION}

We denote the set of BSs in the network as $\mathcal{B}=$ $\left\{B_{1}, B_{2}, \ldots, B_{B}\right\}$, where $B_{1}$ denotes the capacity band of macro BS, $B_{2}$ denotes the coverage band of the macro BS, and $\mathrm{B}_{3}$ to $\mathrm{B}_{\mathrm{B}}$ denote the set of pico BSs. The set of UEs in the network is represented as $\mathcal{S}=\left\{S_{1}, S_{2}, \ldots, S_{S}\right\}$, and the set of supportable power levels of the macro BS is denoted by $\mathcal{P}=\left\{P_{1}, P_{2}, \ldots, P_{P}\right\} . x_{k i}$ and $p_{j}$ are two binary decision variables. $x_{k i}=1$ indicates UE $k$ is served by BS $i$, and 0 otherwise; $p_{j}=1$ indicates the macro cell is using the power level $j$ on capacity band, and 0 otherwise. Let $A$ denote the network coverage with binary elements $a_{k i}$. If UE $k$ is within the coverage of BS $i$, then $a_{k i}=1$; otherwise $a_{k i}=0$. To express the power control in our model, both $A$ and $a_{k i}$ are described as follows:

$$
A=\left[\begin{array}{cccc}
a_{11} & a_{12} & \cdots & a_{1 B} \\
a_{21} & a_{22} & \cdots & a_{2 B} \\
\vdots & \vdots & \ddots & \vdots \\
a_{S 1} & a_{S 2} & \cdots & a_{S B}
\end{array}\right]
$$

and the elements $a_{k i}$ of $A$ are expressed as shown below,

$$
a_{k i}=\left\{\begin{array}{l}
1, \text { if } \sum_{j=c_{k 1}}^{P} p_{j}=1, i=1, \forall k \in \mathcal{S} \\
1, \text { if } 1-\sum_{j=c_{k 1}}^{P} p_{j}=1, i=2, \forall k \in \mathcal{S} \\
1, \text { if } \sum_{j=1}^{c_{k i}} p_{j}=1, \forall k \in \mathcal{S}, \forall i \in\{3, \ldots, B\} \\
0, \text { otherwise, } \forall k \in \mathcal{S}, \forall i \in \mathcal{B}
\end{array}\right.
$$

where $\sum_{j=c_{k 1}}^{P} p_{j}$ is the summation of power level $p_{j}$ from $c_{k 1}$ to the maximal power level $P$, and $c_{k 1}$ means the exact power level when the capacity band of macro cell covers UE $k$. The rationale behind this design is that when we use the power level of capacity band exceeding $c_{k 1}$, UE $k$ is still covered by the macro BS on the same capacity band. Moreover, if UE $k$ cannot be covered by the capacity band of the macro BS, it must be covered by the coverage band of the macro BS. In other words, when $a_{k 1}=0, a_{k 2}=1$, and vice versa. On the other hand, the coverage of pico cells shrinks when the transmit power of the macro cell increases, so we can derive the constraint of pico cells where $c_{k i}$ is the exact power level when UE $k$ is covered by pico BS $i$.

Our problem is described as follows. Given the locations of each user and BSs, we attempt to compute a feasible solution to user association $x_{k i}$, and transmit power level $p_{j}$ to maximize the total utility function of users, which is denoted by $U$.

$\max R_{\text {total }}=\sum_{k \in \mathcal{S}} U\left(R_{k}\right)$

subject to:

$\sum_{i \in \mathcal{B}} x_{k i}=1, \forall k \in \mathcal{S}$,

\section{Algorithm 1: TX power control (TXPC)}

$$
\begin{aligned}
& \text { Initialize capacity band power } P_{c a p} \leftarrow P_{\max }, \text { where } P_{c a p}= \\
& \left\{P_{1}, P_{2}, \ldots, P_{\max }\right\}, \text { Max_Utility } \leftarrow 0 ; \\
& \text { 1. } \text { while }\left(P_{c a p} \neq P_{1}\right) \text { do } \\
& \text { 2. } \quad \text { UEs update association } \\
& \text { 3. } \quad \text { total_utility } \leftarrow \sum_{k=0}^{S} U\left(R_{k}\right) ; \\
& \text { 4. if (total_utility }>\text { Max_Utility) } \\
& \text { 5. } \quad \text { Max_Utility } \leftarrow \text { total_utility; } \\
& \text { 6. } \quad \text { else } \\
& \text { 7. } \quad P_{\text {cap }} \leftarrow P_{c a p+1} ; \\
& \text { 8. } \quad \text { break } \\
& \text { 9. } \quad \text { end if } \\
& \text { 10. } \quad P_{c a p} \leftarrow P_{c a p-1} \\
& \text { 11. end while }
\end{aligned}
$$

Fig. 3. The pseudo code of the proposed TXPC scheme.

$x_{k i} \leq a_{k i}, \forall k \in \mathcal{S}, \forall i \in \mathcal{B}$,

$\sum_{j \in \mathcal{P}} p_{j}=1$,

$x_{k i} \in\{0,1\}, \forall k \in \mathcal{S}, \forall i \in \mathcal{B}$,

$p_{j} \in\{0,1\}, \forall j \in \mathcal{P}$.

(10) states that each UE must be served by one and only one BS, and (11) indicates that an UE can be served by a BS only when the UE is within the coverage of the BS. (12) states that the macro BS can only use one power level on the capacity band at a time. The long-term average throughput $R_{k}$ is as follows:

$$
R_{k}=\sum_{i \in \mathcal{B}} x_{k i}\left[\frac{G\left(Y_{i}\right) r_{k i}}{Y_{i}}\right], \forall k \in \mathcal{S},
$$

where $G\left(Y_{i}\right)$ is the scheduling gain of BSs, and $Y_{i}=$ $\sum_{k \in \mathcal{S}} x_{k i}$ for the number of UEs served by BS $i$. In addition, $r_{k i}$ is the transmission rate of BS $i$ to UE $k$ as described in Sec. II, and is a parameter that corresponds to the transmit power. To express the power control problem in our model, we reformulate (1) as below:

$$
P_{k i}=\Gamma_{k i} \sum_{j \in \mathcal{P}} p_{j} P_{t, j}, \forall k \in \mathcal{S}, \forall i \in \mathcal{B} .
$$

where $P_{t, j}$ is the transmit power corresponding to power level $j$.

Obviously, this problem is a typical nonlinear integer programming problem because $R_{k}$ in the objective function consists of a multiplication of two binary decision variables.

Below, we prove this problem is NP-hard by reduction from the set-packing problem, which is a well-known NP problem.

Definition 1: Given a universe $\mathcal{V}$ and a set $\mathcal{F}$ which consists of some subsets of $\mathcal{V}$, a packing is a subset $\mathcal{G} \subseteq \mathcal{F}$ of sets such that all sets in $\mathcal{G}$ are pairwise disjoint. In the set packing optimization problem, we need to find a set packing that uses the most sets for maximizing the total value.

Theorem 2: The power control and user association problem is NP-hard. 


\section{Algorithm 2: Load-Aware User Association Update} (LAU)

Input: Given the UE set $\mathcal{S}=\left\{s_{1}, s_{2}, \ldots, s_{S}\right\}$, the BS set $\mathcal{B}=\left\{b_{1}, b_{2}, \ldots, b_{B}\right\}$, let $S_{i}=\left\{s_{i 1}, s_{i 2}, \ldots, s_{i N i}\right\} \in \mathcal{S}$ denote the set of users served by BS $i$. where $N_{i}$ is the cardinality of $S_{i}, S I N R_{k i}$ is the SINR of UE $k$ served by BS $i$.

Initialize $i \leftarrow 0$;

1. while $(i \leq \mathrm{B})$

2. $k \leftarrow 0$;

3. while $\left(k \leq N_{i}\right)$

4. $i^{*}=\max _{1<j<B, j \neq i}\left[\frac{G\left(N_{j}+1\right) S I N R_{k j}}{N_{j}+1}\right]$;

5. if $\left[\frac{G\left(N_{i^{*}}\right) S I N R_{k i^{*}}}{N_{i^{*}+1}}\right]>\left[\frac{G\left(N_{i}\right) S I N R_{k i}}{N_{i}}\right]+\varepsilon$

6. $\quad S_{i^{*}} \leftarrow S_{i^{*}}+S_{i k}, S_{i} \leftarrow S_{i}-S_{i k}$;

7. else

8. $\quad k \leftarrow k+1$;

$9 . \quad$ end if

10. end while

11. $i \leftarrow i+1$;

12. end while

Fig. 4. The pseudo code of the proposed LAU scheme

Proof: Our problem is described as follows. The macro BS determines the coverage by adjusting the power level such that the macro BS and pico BSs can serve UEs with the largest transmission rate, thus maximizing the throughput of each set. Given the universe set $\mathcal{V}=\left\{V_{1}, V_{2}, \ldots, V_{M}\right\}$ and $\mathcal{F}=\left\{F_{1}, F_{2}, \ldots, F_{B}\right\}$, let $\mathcal{S}=\left\{V_{1}, V_{2}, \ldots, V_{M}\right\}=\mathcal{V}$. Also, if there is only one power level, we let $A=\left\{F_{1}, F_{2}, \ldots, F_{B}\right\}=$ $\mathcal{F}$, where $A$ denotes the network coverage, and $F_{1}=$ $A_{11}, F_{2}=A_{21}, \ldots, F_{B}=A_{B 1}$. Thus, the set packing problem is a special case of our problem, corresponding to a single power level. Although in this problem, the number of sets remains the same at different power levels, the size of the sets may be changed thus affecting the transmission rate for each UE, and altering the throughput of each set. Therefore, our problem is more difficult as there are more power levels. Since the set-packing problem is a well-known NP problem, it follows that our problem is NP-hard.m

\section{POWER CONTROL AND USER ASSOCIATION MECHANISM}

In this section, we propose a near-optimal heuristic algorithm which determines the power level on the capacity band of macro BS and the user association for two-tier HetNet.

\section{A. Transmit Power Control of Macro BSS}

The Transmit Power Control Algorithm (TXPC) is described as follows. First, the transmit power of the macro BS's capacity band is set to its maximal level. To offload more users from the macro BS to pico BSs, the macro BS reduces the transmit power of its capacity band iteratively. Users then update their associations according to the
Table 1. Simulation Parameters [12]

\begin{tabular}{|c|c|}
\hline Cell Layout & 19-node hexagonal grid \\
\hline Cell Radius & $1.5 \mathrm{~km}$ \\
\hline Bandwidth & $10 \mathrm{MHz}$ \\
\hline Path loss & $\begin{array}{c}\text { Macro to UE: } \mathrm{L}=128.1+37.6 \log 10(\mathrm{R}) \\
\text { Pico to UE: } \mathrm{L}=140.7+36.7 \log 10(\mathrm{R})\end{array}$ \\
\hline Noise Figure & Macro: $5 \mathrm{dBm}$, Pico: $14 \mathrm{dBm}$ \\
\hline Max TX Power & Macro: $43 \mathrm{dBm}$, Pico: $30 \mathrm{dBm}$ \\
\hline $\begin{array}{c}\text { Pico cell } \\
\text { Density }\end{array}$ & 5 per macro cell \\
\hline User Density & 100 per macro cell \\
\hline Scheduler & Proportional Fairness \\
\hline
\end{tabular}

channel conditions and traffic loads of BSs. After the association is updated, the macro BS collects the average data rate from the users and calculates the total system utility function $\sum_{k=1}^{S} \log \overline{R_{k}}$. Finally, the macro $\mathrm{BS}$ determines whether or not to further reduce the transmit power or go back to the previous power level according to the total system utility gain. As the macro BS transmit power is reduced, the SINR of UEs in the macro cell becomes worse and the load of the macro cell decreases, while the SINR of UEs improves, the load of the pico cells increase. Since the capacity is determined by both RSS and the load conditions of BSs, a near-optimal power level can be expected at the end of the iteration. The pseudo code of the proposed TXPC scheme is summarized in Fig. 3.

\section{B. Load-Aware User Association}

In the previous section, traffic offloading can be realized by reducing the transmit power of the macro $\mathrm{BS}$ on the capacity band without degrading the performance of the users at the cell edge. However, traditional RSRP-based user association schemes can be further enhanced when account for the load conditions of BSs since the BSs with lighter load could have the potential to serve more users and enhance total system performance. For this, we propose a new scheme called Load-Aware User Association (LAU) to determine user association based on the expected throughput of UEs instead of the signal strength. In our LAU scheme, each UE estimates the channel condition of its candidate BSs and reports the value to the macro BS. The information combined with the load condition of the BSs is then used to calculate the expected throughput of the user. Finally, macro $\mathrm{BS}$ notes the UE to associate with the $\mathrm{BS}$ which can provide the highest expected throughput. The procedure of LAU is summarized in Fig. 4. Note that if too many users change their associations within a short time, the ping-pong effect may occur. A handover threshold $\varepsilon$ is introduced to prevent such oscillation in user association. By combining with LAU, our power control mechanism can further improve the system utility. 


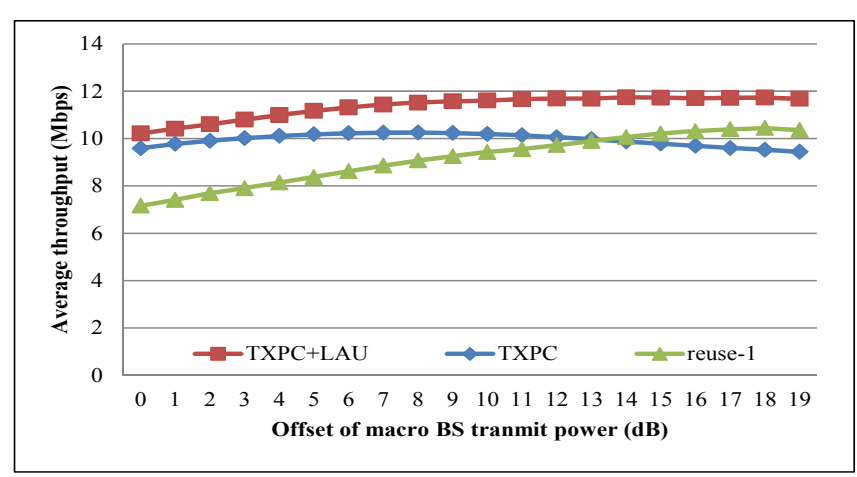

Fig. 5. Average throughput

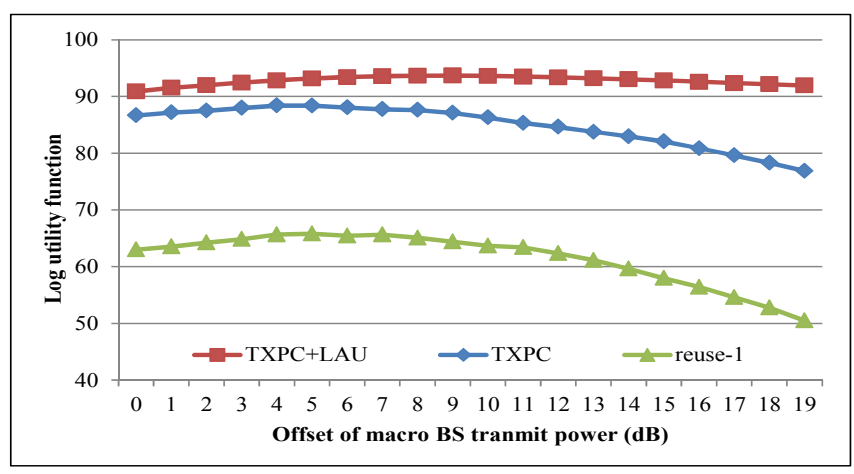

Fig. 6. Total utility function

\section{Complexity Analysis}

Since each UE-BS pair in each power level are chosen at most once, the complexity of our algorithm is $O(\mathrm{~B} \times S \times P)$, where $\mathrm{B}, \mathrm{S}, P$ is the number of BSs, UEs, and the power levels supported by the macro BS. Therefore, our algorithm is indeed a polynomial time algorithm. In practice, the periodicity of TXPC and LAU should balance between the computing/signaling overhead and the accuracy of the algorithm, which is more system independent. Similarly, the user association can be executed actively or only for new arrival and handover process of UEs.

\section{PERFORMANCE EVALUATION}

In this paper, we evaluate the performance of our transmit power control (TXPC) and load-aware user association (LAU) schemes by simulations, and compare them with the general resource allocation scheme.

\section{A. Simulation Setup}

In our simulations, the pico BSs are uniformly distributed in each macro cell. Users are distributed in a hotspot topology, where $50 \%$ of the users are distributed in each pico cell with a radius of $100 \mathrm{~m}$ and the remaining users are uniformly distributed in the macro cell. The total bandwidth is $10 \mathrm{MHz}$ and the ratio of the capacity band and coverage band is set to 1:1. The other parameters follow those in [12], and are summarized in Table 1. We compare our proposed schemes with the general reuse-1 scheme in LTE-A where both the macro cell and pico cells share the whole available bandwidth [7].

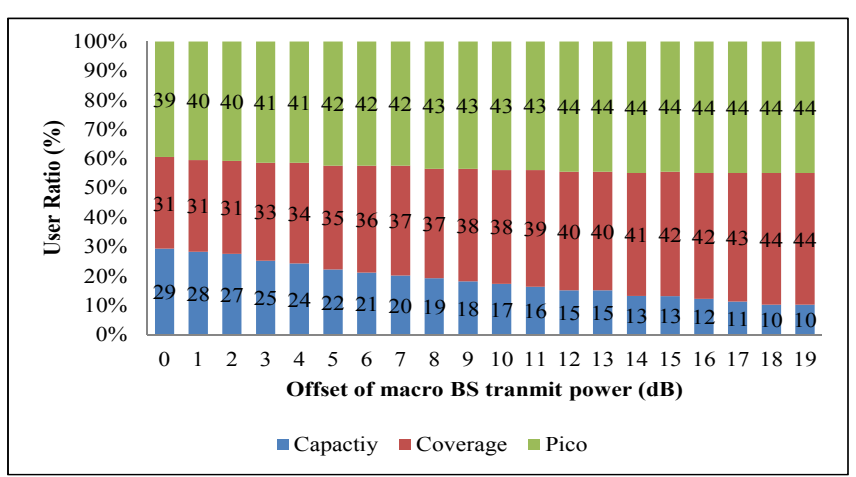

(a) TXPC with LAU

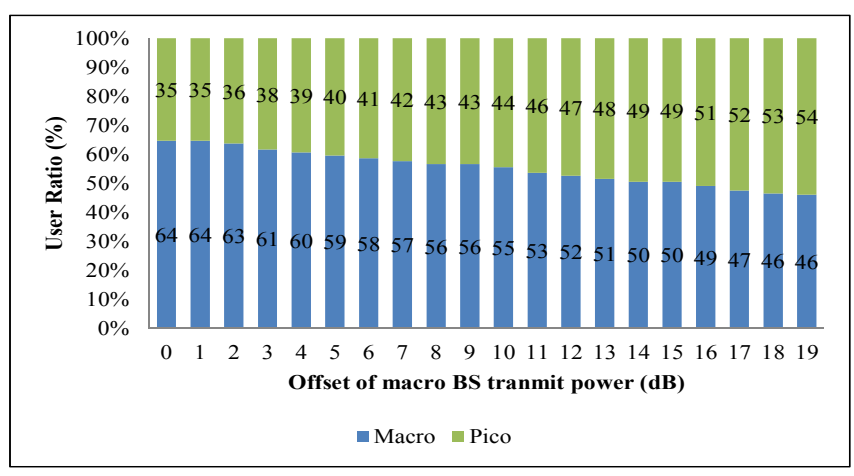

(b) Reuse-1

Fig. 7. User distributions in different schemes

\section{B. Simulation Results}

Fig. 5 shows the average throughput with different reduction of power levels from the maximum transmit power on the capacity band. In TXPC, the macro BS allocates its user only according to the SINR threshold. Note that the power reduction in reuse- 1 decreases the transmit power on all bands, while in our mechanism, only the transmit power on the capacity band is reduced.

We observe that among these methods, there exists an optimal power level. TXPC can achieve this optimal value since it iteratively searches for better total utility. As a result, $43 \%$ and $64 \%$ of gain improvements on average throughput can be achieved by TXPC and TXPC with LAU, respectively, compared with the basic reuse-1 scheme where macro BSs transmit at its maximum power level.

In TXPC, the macro BS maintains the maximum transmit power on the coverage band to protect its edge users, thus causing severe interference to the pico UEs on such band. As can be seen, the average throughput in the TXPC-only scheme is worse than that in the reuse- 1 scheme when the transmit power of the macro BS is low since the average throughput is dominated by pico UEs and pico BSs usually have much lower load. This can be solved by TXPC with LAU since it adjusts the association for different power levels in order to fully utilize the benefit of power control. On the other hand, the macro users in the reuse- 1 scheme may suffer since the poor throughput of the users on macro BS's capacity band is neglected. We will discuss this scenario soon. 
In Fig. 6, our objective is to maximize the total utility function instead of the aggregate throughput. As in [13][14], we choose the $\log$ utility function $\sum_{k=0}^{S} \log \overline{R_{k}}$, where $S$ is the number of the users and $\overline{R_{k}}$ is the average throughput of user $k$, to realize proportional fairness, so as to achieve a balance between system throughput and fairness. TXPC with LAU still has the best performance of these four schemes. More observations in Fig. 6 are described as follows:

(i) The utility in the reuse- 1 scheme is much lower than our proposed scheme as compared to that in Fig. 5. The reason is that as the transmit power is reduced, the users at the edge of the macro cell suffer the poorest SINR if no additional mechanism is provided to protect them.

(ii) The optimal power level is higher than that in Fig. 5 for all schemes. The reason is similar to that in (i): the throughputs of macro users become worse before the maximal aggregate throughput is reached.

(iii) The curve for TXPC with LAU is flatter than that for TXPC-only. Although the SINR of cell edge users is reduced in TXPC, TXPC together with LAU can adapt different transmit power levels according to the load and channel conditions.

The distributions of user association for the reuse-1 scheme and the TXPC with LAU scheme are compared in Fig. 7. In Fig. 7 (a), as the transmit power on the capacity band is reduced, LAU can properly offload the UEs on the capacity band to pico BSs or cover them on the coverage band based on the channel conditions of UEs and the load conditions of BSs. However, in Fig. 7 (b), without the coverage band, the users in the macro cell can only be offloaded to the pico cells when the transmit power of the macro BS is decreased for the reuse- 1 scheme. Although the number of UEs covered by pico BSs is larger than that for the TXPC with LAU scheme, we can infer from Fig. 6 that the cost of offloading is the poor throughput of the UEs with the worst performance, leading to unfairness.

\section{CONCLUSION}

In this paper, we study the offloading issue in a twotier heterogeneous network (HetNet) with one macro BS and several pico BSs. We address the tradeoff between the spectral efficiency and inter-cell interference. We formulate this problem as a nonlinear integer programming problem and propose a novel mechanism which consists of $i$ ) an iterative transmit power control algorithm (TXPC) for macro BS, and ii) a load-aware user association algorithm (LAU) to determine the user association, frequency allocation, and transmit power. Our mechanism jointly considers the channel condition and load information to optimize the network performance. The simulation results show that our algorithm indeed improves network performance in terms of the average throughput and fairness.

\section{ACKNOWLEDGEMENT}

This work was supported in part by the Excellent Research Projects of National Taiwan University, under Grant Number AE00-00-04, in part by the Ministry of Science and Technology (MOST), Taiwan, under Grant Number 1022221-E-002-014-MY2, and in part by the Thematic Research Program of Academia Sinica, under Grant Number 23-24.

\section{REFERENCES}

[1] T.-S. Kim, H. Lim, and J. C. Hou, "Improving Spatial Reuse Through Tuning Transmit Power, Carrier Sense Threshold, and Data Rate in Multihop Wireless Networks," in ACM MobiCom 2006.

[2] A. Damnjanovic, J. Montojo, Y. Wei, T. Ji, T. Luo, M. Vajapeyam, T. Yoo, O. Song, and D. Malladi, "A Survey on 3GPP Heterogeneous Networks," IEEE Wireless Communications, vol.18, no.3, pp.10-21, June 2011

[3] "Small Cells - What's the Big Idea?," white paper, Small Cell Forum, Feb. 2012.

[4] 3GPP "E-UTRA, Radio Resource Control (RRC); Protocol specification", TS 36.331 V11.2.0.

[5] D. Lopez-Perez, X. Chu, and I. Guvenc, "On the Expanded Region of Picocells in Heterogeneous Networks," IEEE Journal of Selected Topics in Signal Processing, vol. 6, no.3, pp. 281 -294, Jun. 2012.

[6] Y. Bejerano and S. Han, "Cell Breathing Techniques for Load Balancing in Wireless LANs," IEEE Transactions on Mobile Computing, vol.8, no.6, pp.735,749, June 2009.

[7] R. Madan, J. Borran, A. Sampath, N. Bhushan, A. Khandekar, and T. $\mathrm{Ji}$, "Cell Association and Interference Coordination in Heterogeneous LTE-A Cellular Networks," IEEE Journal on Selected Areas in Communications (JSAC), vol.28, no.9, pp.1479-1489, Dec. 2010.

[8] Q. Li, R. Q. Hu, Y. Xu, and Y. Qian, "Optimal Fractional Frequency Reuse and Power Control in the Heterogeneous Wireless Networks," IEEE Transactions on Wireless Communications, vol. 12, no. 6, pp. 2658-2668, 2013.

[9] K. Son, S. Chong, and G.Veciana, "Dynamic Association for Load Balancing and Interference Avoidance in Multi-Cell Networks," IEEE Transactions on Wireless Communications, vol. 8, no.7, pp. 35663576, July 2009.

[10]H. Kim, G. de Veciana, X. Yang, and M. Venkatachalam, "Distributed $\propto$-Optimal User Association and Cell Load Balancing in Wireless Networks," IEEE/ACM Transactions on Networking, vol. 20, no.1, pp. 177-190, Feb. 2012.

[11]Q. Ye, B. Rong, Y. Chen, M. Al-Shalash, C. Caramanis, and J.G. Andrews, "User Association for Load Balancing in Heterogeneous Cellular Networks," IEEE Transactions on Wireless Communications, vol.12, no.6, pp.2706,2716, June 2013

[12] 3GPP "Further Advancements for E-UTRA Physical Layer Aspects" TS36.814 v9.0.0.

[13]F. Kelly, "Charging and Rate Control for Elastic Traffic," European Transactions on Telecom, vol. 8, pp. 33-37, Jan./Feb. 1997.

[14]A. Jalali, R. Padovani, and R. Pankaj, "Data Throughput of CDMAHDR: a High Efficiency-High Data Rate Personal Communication Wireless System," in IEEE VTC-Fall 2000 